\title{
Thin Metal Superlens Imaging in Nanolithography
}

\author{
Jing Wang $\mathbb{D}$ and Yunlong Sheng $\mathbb{D}$ \\ Département de Physique, de Génie Physique et d'Optique; Centre d'Optique, Photonique et Laser (COPL), \\ Université Laval, Québec, G1V 0A6, Canada \\ Correspondence should be addressed to Yunlong Sheng; sheng@phy.ulaval.ca
}

Received 31 January 2019; Accepted 26 March 2019; Published 2 May 2019

Academic Editor: John T. Sheridan

Copyright ( 2019 Jing Wang and Yunlong Sheng. This is an open access article distributed under the Creative Commons Attribution License, which permits unrestricted use, distribution, and reproduction in any medium, provided the original work is properly cited.

Superlens imaging system in nanolithography can be regarded as a cascade of two F-P cavities, i.e., a superlens cavity and a dielectric cavity between superlens and introduced mask of high loss, and the transfer function of system is obtained by considering multiple reflections inside the two cavities. For the range of wavevector of interest, the typical high peak of transmission coefficient of superlens coincides with a local minimum of transmission coefficient of dielectric cavity. The peak of transfer function of system corresponds to the peak of transmission coefficient of dielectric cavity. Thin superlens imaging system in nanolithography is analyzed based on transfer function, which can be flattened by simply tuning transmission coefficient of dielectric cavity and superlens cavity. The results are further validated by Finite Element Method (FEM) simulations.

\section{Introduction}

The metal planar superlens, with a negative permittivity at optical frequency and a positive permeability, was proposed by Pendry in 2000 [1] as an alternative to negative index media (NIM) by Veselago in 1967 [2] to break the diffraction limit. The principle of superlens is to compensate the exponential decay of the evanescent field away from the object by amplifying the evanescent waves through surface plasmons (SP) resonances. Since then, many research efforts had been devoted into superlens imaging [3-15], including theoretical models, numerical simulations, experimental demonstrations, and applications.

The silver superlens was demonstrated experimentally with the resolution of one-sixth of the illumination wavelength by Zhang et al. in 2005 [3]. In their experiment, 35 $\mathrm{nm}$ thick silver slab was used because it gives the optimum transfer function. Thinner sliver slabs show higher but narrow enhancement bands, and thicker slabs show smaller enhancements in reference to zero-order transmission [4]. In order to eliminate the sharp peak of transfer function, Sheng et al. proposed designing the metallic superlens close to the cutoff condition of the long-range SP mode to balance the amplification by the SP resonance and the flatness of the transfer function $[5,6]$. Moore et al. suggested a performance window for superlens with total thickness range from 120 $\mathrm{nm}$ to $140 \mathrm{~nm}$ in order to get a flatter transfer function [7]. Those were reported by considering transfer functions of the imaging system with three-layer structure, a superlens sandwiched by two semi-infinite dielectrics [4-7].

Normally, when dealing with the imaging system, a perfectly absorbing, thin screen with slit(s) has been widely adopted. The transmission of the idealized screen will be 1 in the slit or 0 otherwise. While in real nanolithography applications, there exists a metallic object mask. The metallic mask itself exists mainly for constructing a perfect object function in the object plane just behind the mask for the imaging system. To obtain a good enough object function, which means as close as the ideal case, i.e., 1 in the slit and 0 otherwise, a thick mask with intrinsic high loss, such as Chrome $(\mathrm{Cr})$, is usually applied. Due to the introduction of mask, a dielectric cavity is naturally formed between the mask and superlens. Blaikie et al. approximately considered the neglected recursive reflections in the dielectric cavity between the superlens and the mask and modified transfer function by an improved transfer-matrix model [8]. Sheng et al. further took possible SPR by mask itself in some cases into account and gave a more general model with its transfer function, which is optimized by genetic algorithm (GA) [9].

For the superlens imaging system in nanolithography, we regarded it as two cascaded F-P cavities, a superlens cavity 


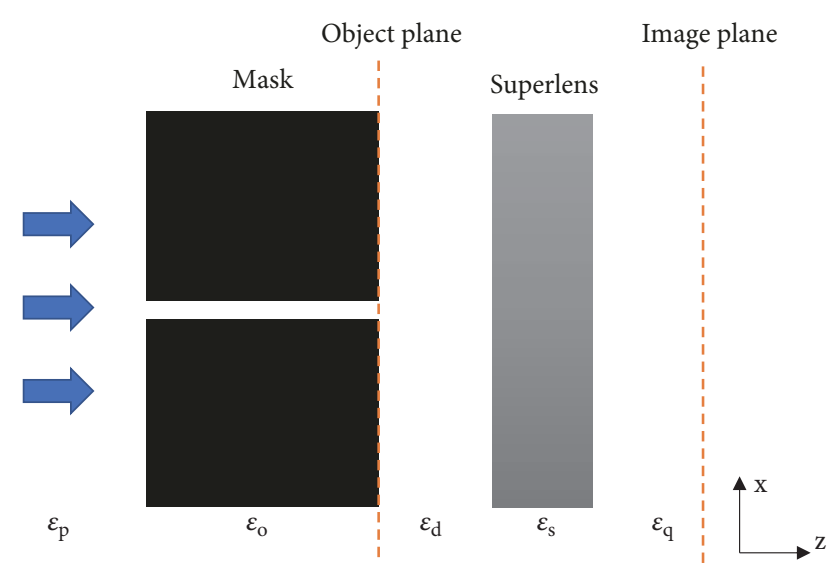

FIGURE 1: Metal superlens imaging system for nanolithography.

and a dielectric cavity between superlens and introduced mask of high loss, and the transfer function of system is obtained by considering multiple reflections inside the two cavities $[9,10]$. We studied the transfer function of system and the transmission coefficient of two cavities and then revealed some relations among them. It is found that the peak shown in transmission coefficient of superlens always corresponds to the local minimum of transmission coefficient of dielectric cavity. Moreover, the peak of transfer function of system coincides with transmission coefficient of dielectric cavity instead of that of superlens. Based on these, we propose that, by simply tuning the transmission coefficient of superlens cavity and dielectric cavity, a very thin metal superlens imaging system can show well-balanced transfer function and thus produces improved image, which is validated by FEM simulations.

\section{Metal Superlens Imaging System}

Consider an imaging system for nanolithography with a metal superlens of permittivity $\varepsilon_{s}$ and a thick metallic mask of permittivity $\varepsilon_{o}$ with high intrinsic loss to construct an object function, as shown in Figure 1. For the purpose of the image analysis, herein a slit of nanosize is perforated through the object mask. A dielectric cavity, with permittivity $\varepsilon_{d}$, is formed between the superlens and the mask. The illuminating light of TM polarization is incident normally to the object mask from the semi-infinite dielectric medium $\varepsilon_{p}$. The image plane is placed in a semi-infinite dielectric medium $\varepsilon_{q}$ at a distance $d_{q}$ from the superlens. The incident plane wave of TM polarization was launched in dielectric medium $\varepsilon_{p}$ and then encountered the object mask $\varepsilon_{o}$. In the case where the lithographic metal mask layer is thick and of high intrinsic loss, the incident waves will be dissipated in the mask medium and can only pass through the slit. At the exit of the slit, the waves, labelled as source A, will be scattered in the medium $\varepsilon_{d}$ launching the Surface Plasmon Polaritons (SPPs) along interfaces of the superlens. Both the homogeneous propagating waves and inhomogeneous SPP waves will reach the image plane and contribute to the image in dielectric medium $\varepsilon_{q}$. The evanescent waves will simply undergo an exponential delay in the semi-infinite dielectric medium $\varepsilon_{q}$. Therefore, the imaging system for source A shown in Figure 1 can be regarded as two cascaded Fabry-Perot (F-P) cavities. The first cavity is the dielectric layer $\varepsilon_{d}$ with two metal/dielectric interfaces, $\varepsilon_{d} / \varepsilon_{o}$ and $\varepsilon_{d} / \varepsilon_{\mathcal{s}}$, respectively. The second cavity is the metal superlens layer with two interfaces, $\varepsilon_{s} / \varepsilon_{d}$ and $\varepsilon_{s} / \varepsilon_{q}$, respectively. The transfer function of the imaging system can be computed as the product of transmission coefficients of the two cavities, which are obtained by considering multiple reflections inside the two cavities. We analyze the mathematical expressions of the transmission coefficients corresponding to the two cavities in order to get a flat transfer function of the imaging system.

2.1. Transmission Coefficient of Superlens Cavity. The superlens cavity of permittivity $\varepsilon_{s}$ is between two metal/dielectric interfaces of $\varepsilon_{s} / \varepsilon_{d}$ and $\varepsilon_{s} / \varepsilon_{q}$. Its transmission coefficient can be calculated by considering resonance of multiple reflected fields in the superlens cavity as $[1,5]$

$$
\tau_{s}=\frac{e_{s} t_{d s} t_{s q}}{1-e_{s}^{2} r_{s d} r_{s q}}
$$

where the Fresnel reflection and transmission coefficients from medium $i$ to medium $j$, with subindices $i, j=o, d$, $s$, and $q$, are $r_{i j}=\left(\varepsilon_{j} k_{z i}-\varepsilon_{i} k_{z j}\right) /\left(\varepsilon_{j} k_{z i}+\varepsilon_{i} k_{z j}\right)$ and $t_{i j}=$ $2 \varepsilon_{j} k_{z i} /\left(\varepsilon_{j} k_{z i}+\varepsilon_{i} k_{z j}\right)$ with $k_{z i}=\sqrt{\varepsilon_{i} k_{0}^{2}-k_{x}^{2}}$; the propagation factor $e_{i}=\exp \left(i k_{z i} d_{i}\right)$, describing the phase change of the propagating waves with $k_{x}^{2}<\varepsilon_{i} k_{0}^{2}$ along the distance $d_{i}$ and the exponent decay in amplitude of the evanescent waves $k_{x}^{2}>\varepsilon_{i} k_{0}^{2}$ over $d_{i}$, respectively.

According to (1) and for a silver superlens with variable thickness, the amplitude of transmission coefficient $\left|\tau_{s}\right|$ as a function of normalized wavevector $k_{x 0}=k_{x} /\left(\sqrt{\varepsilon_{d}} k_{0}\right)$ is depicted in Figure 2(a). Typically for a thin superlens of less than $30 \mathrm{~nm}$ in thickness a sharp and high peak is shown at a low spatial frequency $k_{x 01}$ slightly larger than $k_{x 0}=1$ and a relative broad and low peak is at a high spatial frequency $k_{x 02}$. When increasing the thickness only one peak will appear at a spatial frequency between spatial frequency $k_{x 01}$ and $k_{x 02}$. For large thickness of superlens, the amplitude of transmission coefficient becomes low and flat due to high loss in the thick metal superlens, as shown in Figure 3(a).

According to Maxwell's equations and the boundary conditions in the Dielectric-Metal-Dielectric (DMD) waveguide structure, the dispersion relation can be obtained and solved numerically [5]. The effective indices of waveguide modes are plotted for different thickness of Ag superlens in Figure 2(b). Two modes, long-range surface plasmon (LRSP) mode and short-range surface plasmon (SRSP) mode, are supported for DMD waveguide of a thin superlens. The two peaks correspond to such two excited SP modes: a LRSP mode associated with a narrow peak located at lower spatial frequencies $k_{x 01}$ and a SRSP mode associated with a broad peak at higher spatial frequencies $k_{x 02}$. When the thickness of the metal superlens increases, location of the peak at low frequency (LRSP mode) tends to shift to higher frequency, and location of the peak at high frequency (SRSP mode) tends 


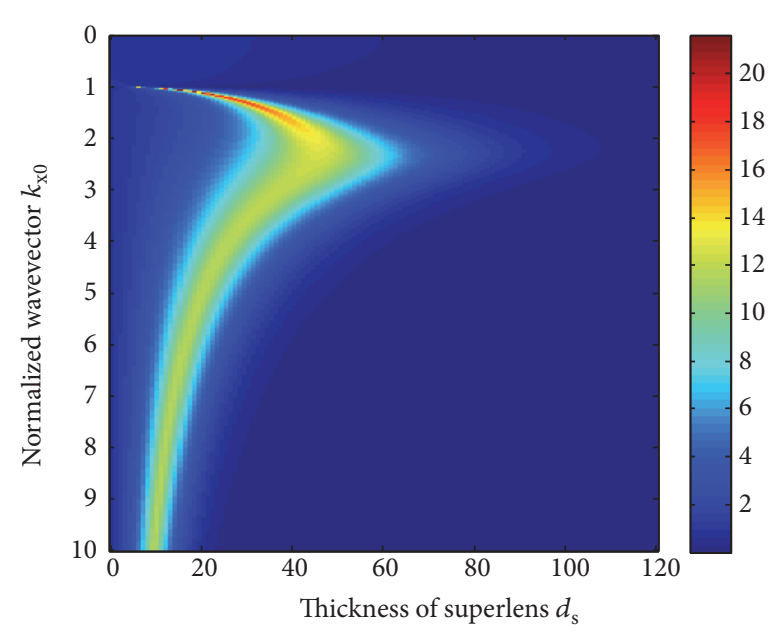

(a)

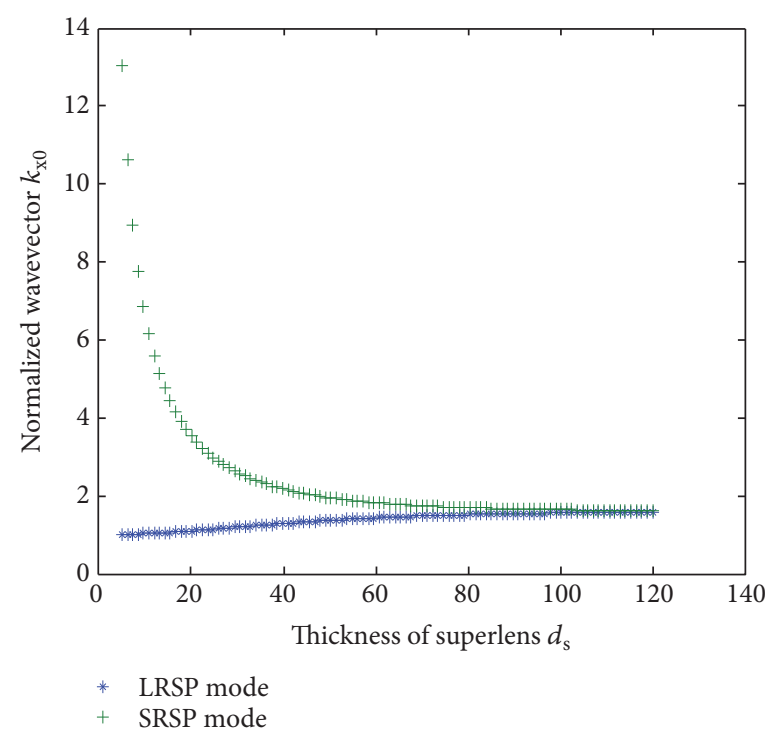

(b)

FIGURE 2: For Ag superlens of thickness ranging from $5 \mathrm{~nm}$ to $120 \mathrm{~nm}$ sandwiched by two dielectric mediums $\varepsilon_{d}=\varepsilon_{q}=2.34$, (a) the amplitude of transmission coefficient $\left|\tau_{s}\right|<1$ for propagating waves with $k_{x 0}<1$, while for evanescent waves with $k_{x 0}>1$, it can be amplified. (b) Mode effective index of DMD waveguide.

to lower frequency. At a certain thickness of the superlens, only one mode will be supported, which accounts for only one peak of the transmission coefficient.

If the imaging system is considered as a superlens between two semi-infinite dielectrics without presence of the metal lithographic mask, as that in [4-7], then the very thin superlens is usually inappropriate for imaging due to the existence of sharp peak of $\left|\tau_{s}\right|$ resulting from excitation of
LRSP mode. The LRSP mode can be cutoff [5] if $r_{d s}+e_{s}^{2} r_{s q} \approx 0$ is met, which means the reflection of metal layer is around 0 .

2.2. Transmission Coefficient of Dielectric Cavity. The dielectric slab of $\varepsilon_{d}$ is sandwiched by the metal superlens and the metal mask, constituting a dielectric F-P cavity. Its transmission coefficient can be calculated $[9,10]$ by taking into account the recursive reflections in the cavity as

$$
\tau_{d}=\frac{e_{d}}{1-e_{d}^{2}\left(\left(r_{d s}+e_{s}^{2} r_{s q}\right) /\left(1-e_{s}^{2} r_{s d} r_{s q}\right)\right)\left(\left(r_{d o}+e_{o}^{2} r_{o p}\right) /\left(1-e_{o}^{2} r_{o p} r_{o d}\right)\right)} .
$$

For a thick mask with high loss used in the superlens image system for nanolithography, $r_{o p}=0$; then (2) can be further written as

$$
\begin{aligned}
\tau_{d} & =\frac{1}{1 / e_{d}-e_{d}\left(\left(r_{d s}+e_{s}^{2} r_{s q}\right) /\left(1-e_{s}^{2} r_{s d} r_{s q}\right)\right) r_{d o}} \\
& =\frac{1}{1 / e_{d}-\left(\left(r_{d s}+e_{s}^{2} r_{s q}\right) r_{d o} e_{d} /\left(e_{s} t_{d s} t_{s q}\right)\right) \tau_{s}} .
\end{aligned}
$$

For evanescent waves with $k_{x}^{2}>\varepsilon_{d} k_{0}^{2}, e_{d}=$ $\exp \left(i \sqrt{\varepsilon_{d} k_{0}^{2}-k_{x}^{2}} d_{d}\right)=\exp \left(-\sqrt{k_{x}^{2}-\varepsilon_{d} k_{0}^{2}} d_{d}\right)$ is real. The denominator in (3) can be evaluated, based on ||$a|-| b|| \leq|a \pm b| \leq|a|+|b|$ and $|a b|=|a||b|$, as

$$
\begin{aligned}
& \| \frac{\left(r_{d s}+e_{s}^{2} r_{s q}\right) r_{d o} e_{d}}{e_{s} t_{d s} t_{s q}}|| \tau_{s}\left|-\frac{1}{e_{d}}\right| \\
& \leq\left|\frac{1}{e_{d}}-\frac{\left(r_{d s}+e_{s}^{2} r_{s q}\right) r_{d o} e_{d}}{e_{s} t_{d s} t_{s q}} \tau_{s}\right| \\
& \quad \leq\left|\frac{\left(r_{d s}+e_{s}^{2} r_{s q}\right) r_{d o} e_{d}}{e_{s} t_{d s} t_{s q}}\right|\left|\tau_{s}\right|+\frac{1}{e_{d}} .
\end{aligned}
$$

Thus, when the amplitude of transmission coefficient of the superlens $\left|\tau_{s}\right|$ shows a very high peak associated with the excitation of LRSP mode at a low spatial frequency $k_{x 01}$ slightly larger than $k_{x 0}=1$, the amplitude of transmission coefficient of the dielectric cavity $\left|\tau_{d}\right|$ will fall to a local minimum. Meanwhile, $\left|\tau_{d}\right|$ will reach a maximum when the absolute value of denominator of (3) approaches 0 ; i.e., 


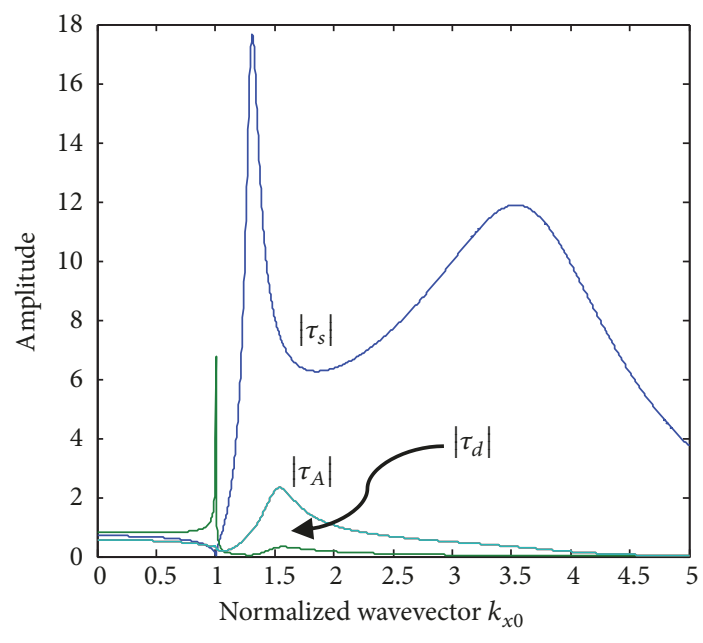

(a)

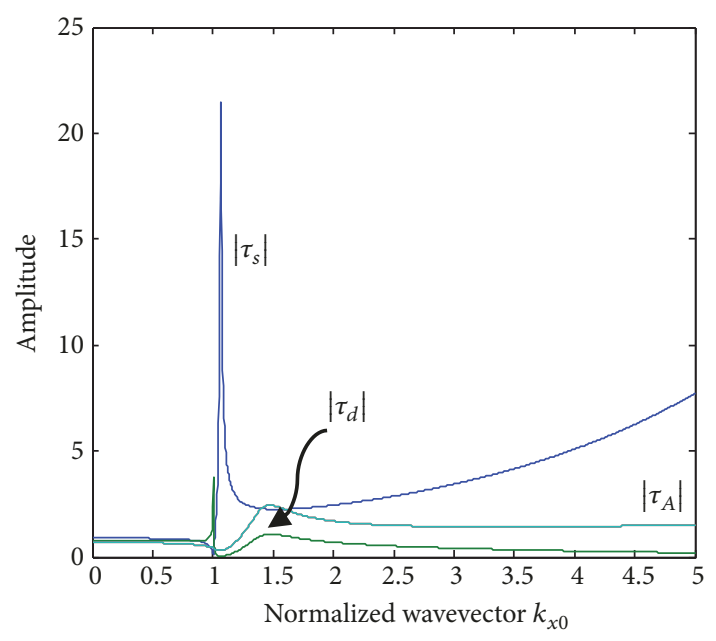

(b)

Figure 3: The amplitude of transmission efficient of superlens and dielectric and transfer function of the system as a function of normalized wavevector. (a) $d_{d}=40 \mathrm{~nm}, d_{s}=30 \mathrm{~nm}$, (b) $d_{d}=10 \mathrm{~nm}$, and $d_{s}=15 \mathrm{~nm}$.

$$
\left|\frac{1}{e_{d}}-\frac{\left(r_{d s}+e_{s}^{2} r_{s q}\right) r_{d o} e_{d}}{e_{s} t_{d s} t_{s q}} \tau_{s}\right| \longrightarrow 0
$$

Besides, it is interesting to notice that if $r_{d s}+e_{s}^{2} r_{s q} \approx 0$ in (3), then the transmission coefficient $\tau_{d} \approx e_{d}$, implying an exponential decay of the evanescent waves in the dielectric cavity, just as that in the free space without any resonances, and is independent of the cavity thickness.

2.3. Transfer Function of Imaging System. As the imaging system for source A is a cascade of two F-P cavities, the total transfer function $\tau_{A}$ is calculated by

$$
\begin{aligned}
\tau_{A} & =\tau_{d} \cdot \tau_{s} \\
& =\frac{1}{1 /\left(e_{d} \tau_{s}\right)-\left(r_{d s}+e_{s}^{2} r_{s q}\right) r_{d o} e_{d} /\left(e_{s} t_{d s} t_{s q}\right)},
\end{aligned}
$$

and when condition (5) is satisfied, $\left|\tau_{A}\right|$ and $\left|\tau_{d}\right|$ will both reach their maximums, according to (3) and (6), at the same spatial frequency $k_{x 03}$. This reveals that the peak of the total transfer function $\left|\tau_{A}\right|$ for source $A$ in the imaging system corresponds to the peak of transmission coefficient of dielectric cavity $\left|\tau_{d}\right|$ at $k_{x 03}$ instead of that of superlens $\left|\tau_{s}\right|$ at $k_{x 01}$. Actually, the value of $\left|\tau_{A}\right|$ at $k_{x 01}$ is always balanced as the transmission coefficient of the superlens $\left|\tau_{s}\right|$ can be suppressed by the local minimum of $\left|\tau_{d}\right|$ at $k_{x 01}$. The transfer function for source $\mathrm{A}$ is defined as the transmission coefficients of the system described in (6) as a function of the spatial spectral frequency. According to SPP waveguide theory, the LRSP mode is cutoff when the propagation constant $\beta$ is purely real, which occurs when the nature of the mode changes from attenuating $(\operatorname{Im}[\beta]>0)$ to growing $(\operatorname{Im}[\beta]<0)$ with the propagation along the metal layer $[5,6]$. If the cutoff condition for LRSP mode, i.e., $r_{d s}+e_{s}^{2} r_{s q} \approx 0$, is fulfilled, the transfer function $\tau_{A}=$ $\tau_{d} \cdot \tau_{s} \approx e_{d} \cdot \tau_{s}$. In this case, a relative flat transfer function can be expected when considering together the well-controlled, proper amplification of SPP by superlens and an exponential decay of amplitude of evanescent waves in dielectric cavity.

\section{Imaging by Thin Superlens Imaging System}

We first consider an Ag superlens imaging system with typical parameters: the metallic mask is chrome with permittivity $\varepsilon_{o}=-8.55+i 8.96$ and thickness of $d_{o}=50 \mathrm{~nm}$ and the silver slab with permittivity $\varepsilon_{s}=-2.6+i 0.25$ with incident wavelength of $365 \mathrm{~nm}$ and thickness $d_{s}=30 \mathrm{~nm}$; the dielectric layer between superlens and mask has the permittivity $\varepsilon_{d}=$ 2.34 and thickness of $d_{d}=40 \mathrm{~nm}$, and the dielectric $\varepsilon_{q}=2.34$, as shown in Figure 1. From Figure 3(a), the sharp peak of $\left|\tau_{s}\right|$ corresponds to the local minimum of $\left|\tau_{d}\right|$ at $k_{x 01} \approx 1.3$, which is the effective index of LRSP mode excited in DMD waveguide. The broad peak of $\left|\tau_{s}\right|$ at $k_{x 02} \approx 3.6$ is shown because of the excitation of SRSP mode. The value of transfer function $\left|\tau_{A}\right|$ at $k_{x 01} \approx 1.3$ is balanced by the multiplication of $\left|\tau_{s}\right|$ and $\left|\tau_{d}\right|$, which indicates that we cannot judge the imaging system only by transmission coefficient of superlens. The peak of transfer function $\left|\tau_{A}\right|$ locates at $k_{x 03} \approx 1.6$, which is the same as that of transmission coefficient of dielectric cavity $\left|\tau_{d}\right|$. We change the thickness of Ag to $d_{s}=15 \mathrm{~nm}$ and thickness of dielectric $d_{d}=10 \mathrm{~nm}$, which form a thinner superlens imaging system. The transmission coefficient $\left|\tau_{s}\right|$ of $15 \mathrm{~nm}$ thick superlens has a shaper and higher peak, as shown in Figure 3(b), than that of superlens of $30 \mathrm{~nm}$ thickness in Figure 3(a). Still, the peak of $\left|\tau_{s}\right|$ corresponds to the local minimum of $\left|\tau_{d}\right|$ at $k_{x 01} \approx 1.1$, and thus the value of $\left|\tau_{A}\right|$ at $k_{x 01} \approx 1.1$ is balanced in the same manner. The peak of transfer function $\left|\tau_{A}\right|$ and transmission coefficient of the dielectric cavity $\left|\tau_{d}\right|$ is obtained around $k_{x 03} \approx 1.4$, as depicted 


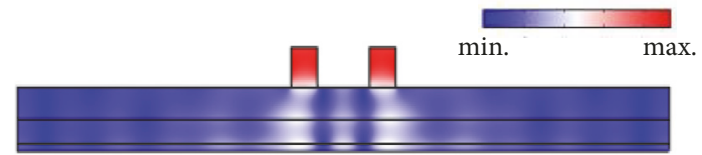

(a)

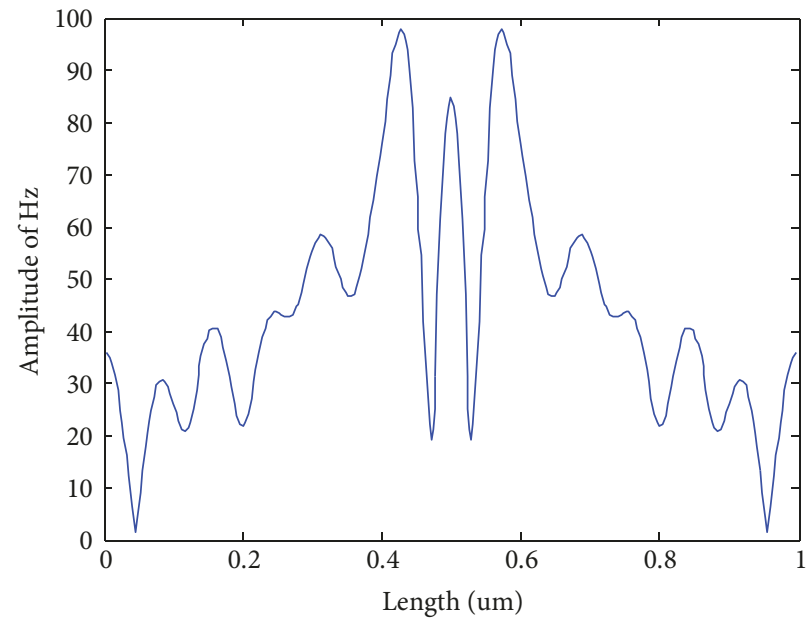

(b)

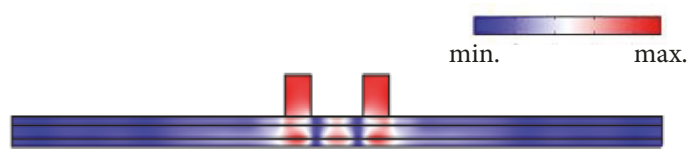

(c)

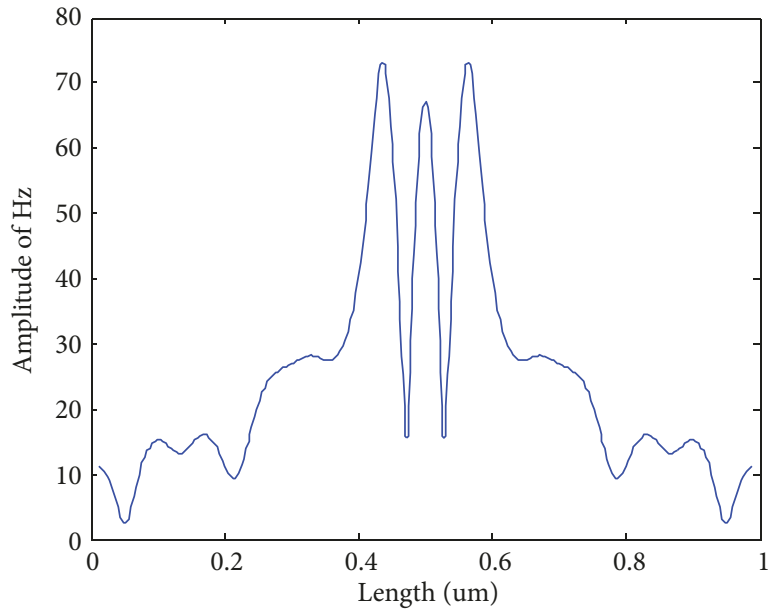

(d)

FIGURE 4: Simulation results of the superlens imaging system by FEM. (a) Hz field distributions of system with $d_{d}=40 \mathrm{~nm}$ and $d_{s}=30 \mathrm{~nm}$; (b) image of two-slit object in the imaging plane in (a); (c) Hz field distributions of system with $d_{d}=10 \mathrm{~nm}$ and $d_{s}=15 \mathrm{~nm}$; (d) image of two-slit object in the imaging plane in (c).

in Figure 3(b). The very thin superlens imaging system can show a flatter transfer function over a broader range of spatial frequency by simply tuning the transmission coefficient of superlens cavity and dielectric cavity.

For further validations, 2D Finite Element Method (FEM) by commercial software package COMSOL Multiphysics 4.4 was employed to simulate the imaging system in $(\mathrm{x}, \mathrm{y})$ plane, as shown in Figure 4. A TM $\left(\mathrm{E}_{\mathrm{x}}, \mathrm{E}_{\mathrm{y}}, \mathrm{H}_{\mathrm{z}}\right)$ plane wave with wavelength of $365 \mathrm{~nm}$ was launched along $\mathrm{y}$ direction from the input port on the top boundary in the FEM simulations. A perfectly matched layer (PML) was applied at the bottom boundary and periodical boundary conditions (PBC) were imposed on the left and right sides. A chrome mask with two slits of $40 \mathrm{~nm}$ was used. The centre-to-centre distance of two slits was $120 \mathrm{~nm}$. The lateral length along $\mathrm{x}$ direction of each layer was $1 \mathrm{um}$. To clearly show the $\mathrm{Hz}$ fields after the mask of high loss, the incident waves before and in the mask and the mask itself are removed in Figures 4(a) and 4(c). The image of the two spaced slits is recorded at the imaging plane placed at a distance of $10 \mathrm{~nm}$ away from the superlens, as shown in Figures 4(b) and 4(d). Obviously, the image in Figure 4(d) by thin superlens system with transfer function in Figure 3(b) is better than the one in Figure 4(b) with the transfer function shown in Figure 3(a), because the side lobes of the image in Figure 4(d) were largely suppressed compared with that in Figure 4(b). However, the amplitude of the unwanted centre peak is still high, as shown in Figures 4(b) and 4(d).

The imaging performance of thin superlens imaging system can be further improved by approaching the LRSP mode cutoff condition as we demonstrated. Change the dielectric $\varepsilon_{d}=2.34$ to $\varepsilon_{d}=4.08$, and keep all others unchanged as those in Figure 4(c). The transfer function $\left|\tau_{A}\right|$ and transmission coefficient $\left|\tau_{d}\right|$ and $\left|\tau_{s}\right|$ are plotted in Figure 5(a), from which we can see that the high peak of $\left|\tau_{s}\right|$ associated with LRSP mode is mainly removed, and $\left|\tau_{d}\right|$ approximately follows an exponential decay for evanescent waves, and thus the transfer function $\left|\tau_{A}\right|$ is balanced to show flatness. The $\mathrm{Hz}$ field by a FEM simulation in this superlens system is shown in Figure 5(b) and the image is notably improved as shown in Figure 5(c), in which both the side lobes and the centre peak are greatly suppressed compared with those in Figures 4(b) and $4(\mathrm{~d})$.

\section{Conclusions}

We investigated the imaging performance of the metal planar superlens for nanolithography, considering the superlens imaging system as a cascade of two F-P cavities: a superlens cavity and a dielectric cavity between superlens and introduced mask of high loss. By analyzing the transmission coefficient of superlens and dielectric cavity and the transfer function of whole system, we found that the peak of the transmission coefficient of the superlens always coincides with the local minimum of that of the dielectric cavity, and the peak of the whole transfer function of system actually corresponds to that of dielectric cavity instead of superlens. Then, we show that very thin superlens imaging system, which was usually believed to be improper for superlens imaging, has an improved imaging performance by simply tuning the transmission coefficient of superlens cavity and dielectric cavity. All the results are analyzed based on transfer functions and further confirmed by FEM simulations. 


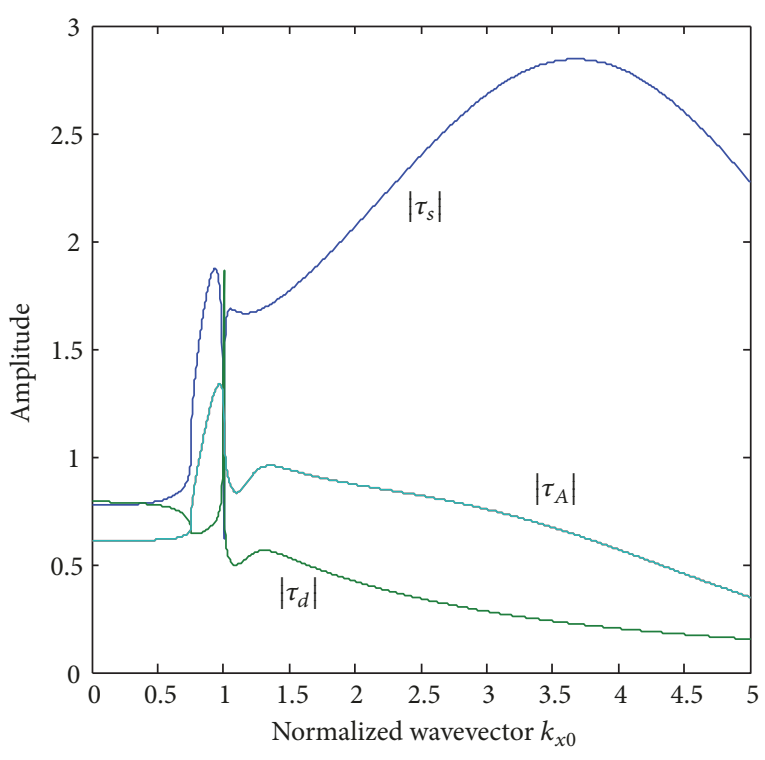

(a)

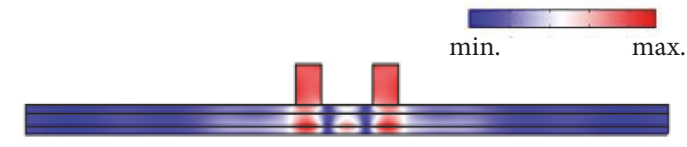

(b)

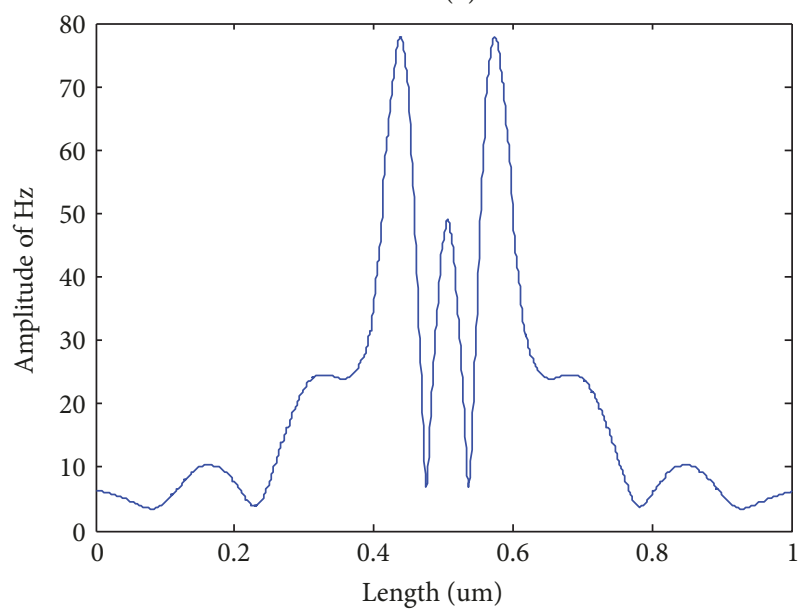

(c)

FIGURE 5: (a) Amplitude of transmission coefficient of superlens and dielectric and transfer function of the system as a function of normalized wavevector. (a) LRSP mode cut-off condition is approached with $d_{d}=10 \mathrm{~nm}, d_{s}=15 \mathrm{~nm}$, and $\varepsilon_{d}=4.08$; (b) Hz field distributions in superlens imaging system; (c) image of two-slit object in the imaging plane in (b).

\section{Data Availability}

The data used to support the findings of this study are included within the article.

\section{Conflicts of Interest}

The authors declare that there are no conflicts of interest regarding the publication of this paper.

\section{Acknowledgments}

The authors acknowledge the support from the Natural Science and Engineering Research Council (NSERC) of Canada, Discovery Grant, to this research program.

\section{References}

[1] J. B. Pendry, "Negative refraction makes a perfect lens," Physical Review Letters, vol. 85, no. 18, pp. 3966-3969, 2000.

[2] V. G. Veselago, "The electromagnetics of substances with simultaneously negative values of epsilon and mu," Soviet Physics Uspekhi, vol. 10, no. 4, pp. 509-514, 1968.

[3] N. Fang, H. Lee, C. Sun, and X. Zhang, "Sub-diffraction-limited optical imaging with a silver superlens," Science, vol. 308, no. 5721, pp. 534-537, 2005.

[4] H. Lee, Y. Xiong, N. Fang et al., "Realization of optical superlens imaging below the diffraction limit," New Journal of Physics, vol. 7, no. 255, 2005.

[5] G. Tremblay and Y. Sheng, "Improving imaging performance of a metallic superlens using the long-range surface plasmon polariton mode cutoff technique," Applied Optics, vol. 49, no. 7, pp. A36-A41, 2010.

[6] G. Tremblay and Y. Sheng, "Designing the metallic superlens close to the cutoff of the long-range mode," Optics Express, vol. 18, no. 2, pp. 740-745, 2010.

[7] C. P. Moore and R. J. Blaikie, "Robust design of a silver-dielectric near-field superlens for photolithography," Journal of the Optical Society of America B: Optical Physics, vol. 30, no. 12, pp. 32723277, 2013.

[8] C. P. Moore, R. J. Blaikie, and M. D. Arnold, "An improved transfer-matrix model for optical superlenses," Optics Express, vol. 17, no. 16, pp. 14260-14269, 2009.

[9] G. Tremblay and Y. Sheng, "Modeling and designing metallic superlens with metallic objects," Optics Express, vol. 19, no. 21, pp. 20634-20641, 2011.

[10] F. Xu, G. Chen, C. Wang, B. Cao, and Y. Lou, "Superlens imaging with a surface plasmon polariton cavity in imaging space," Optics Expresss, vol. 38, no. 19, pp. 3819-3822, 2013.

[11] B. Zhang and J. B. Khurgin, "Eigen mode approach to the subwavelength imaging with surface plasmon polaritons," Applied Physics Letters, vol. 98, no. 26, p. 263102, 2011.

[12] X. Zhang and Z. Liu, "Superlenses to overcome the diffraction limit," Nature Materials, vol. 7, no. 6, pp. 435-441, 2008.

[13] S. Kawata, Y. Inouye, and P. Verma, "Plasmonics for near-field nano-imaging and superlensing," Nature Photonics, vol. 3, no. 7, pp. 388-394, 2009.

[14] C. Wang, W. Zhang, Z. Zhao et al., "Plasmonic structures, materials and lenses for optical lithography beyond the diffraction limit: a review," Micromachines, vol. 7, no. 7, p. 118, 2016.

[15] K. A. Willets, A. J. Wilson, V. Sundaresan, and P. B. Joshi, "Super-resolution imaging and plasmonics," Chemical Reviews, vol. 117, no. 11, pp. 7538-7582, 2017. 

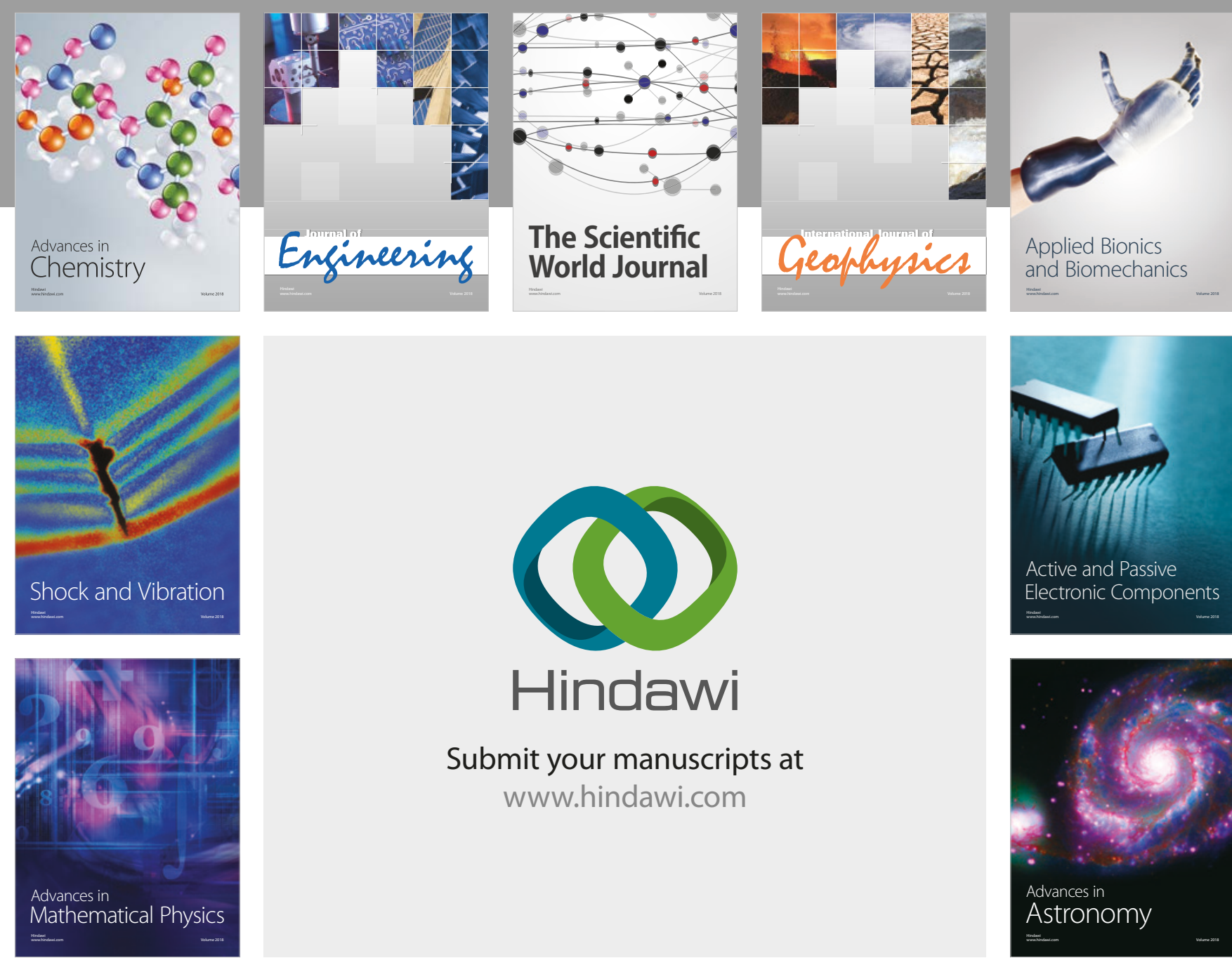

Submit your manuscripts at

www.hindawi.com

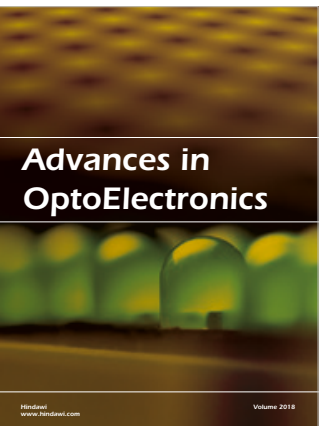

\section{Rotcting Machinery}
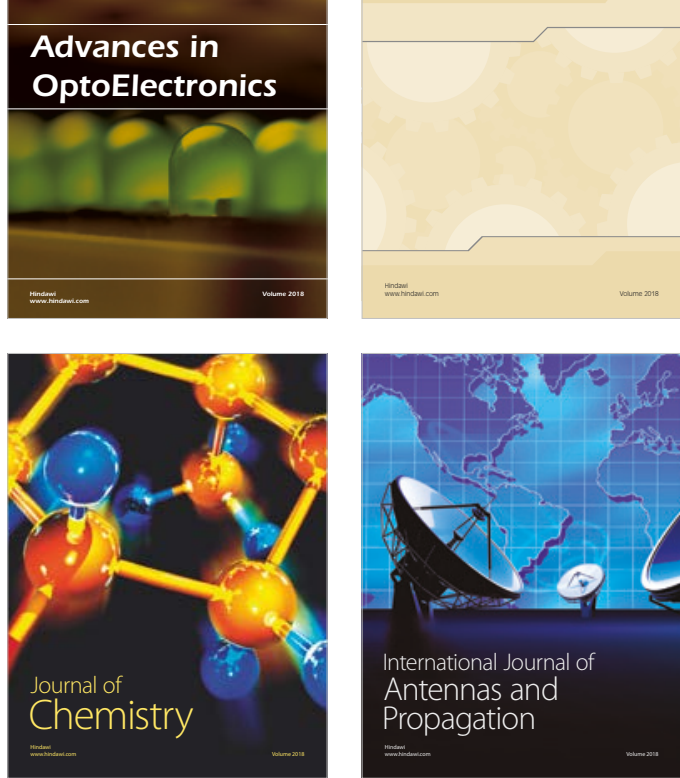

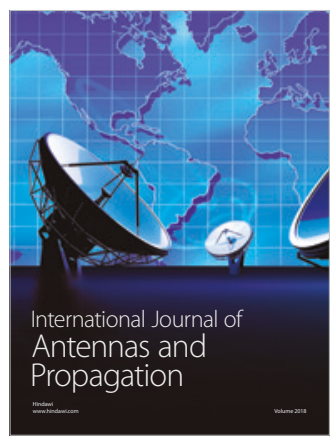

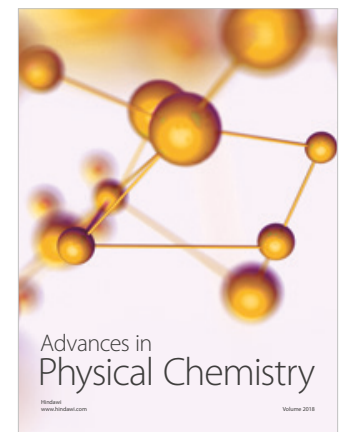

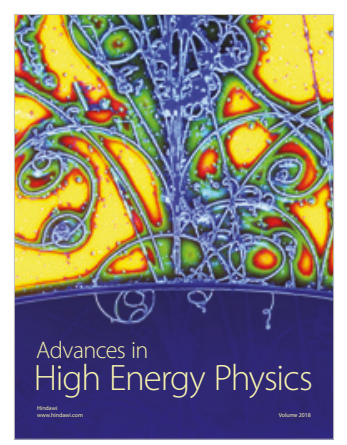

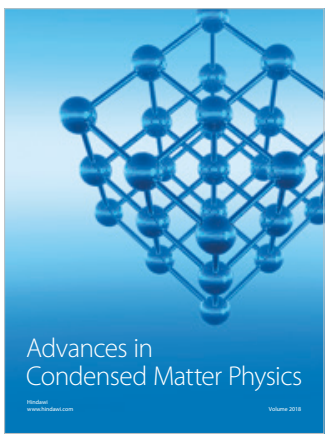

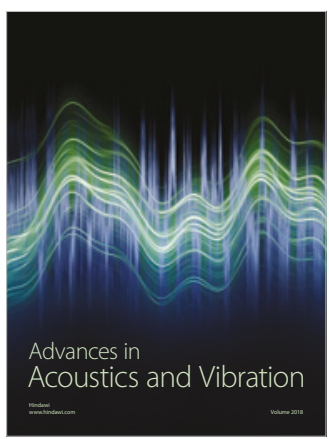

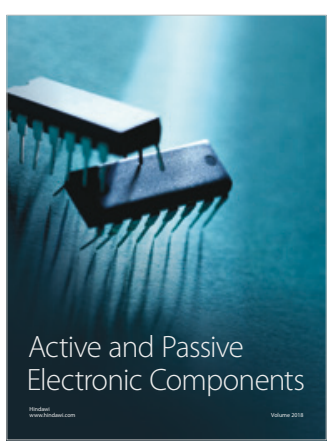
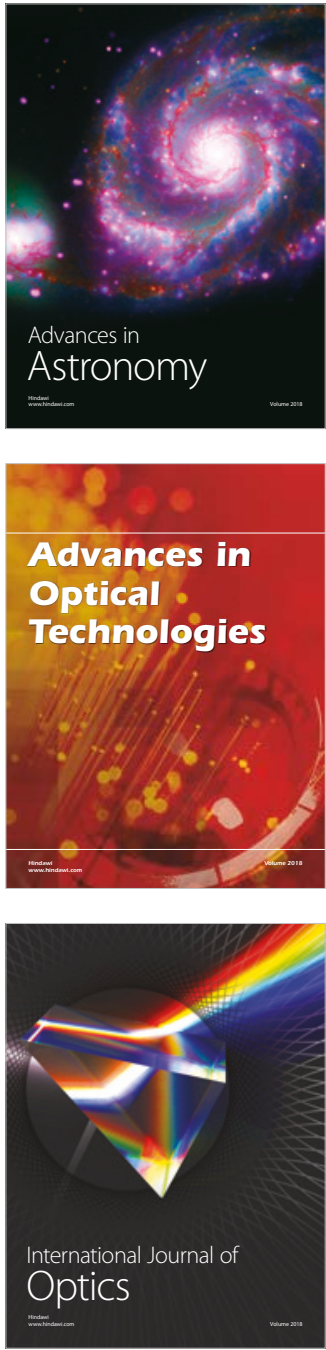